\title{
Errata
}

\section{Free Boundary Optimization-a Constructive Iterative Method}

By Andrew Acker, Z. angew. Math. Phys. 30, 885-900 (1979)

1. Throughout [1],o( $\sigma)$ is used to denote an arbitrary function such that $o(\sigma) \rightarrow 0$ as $\sigma \rightarrow 0$. This definition is at variance with common usage, which requires that $(o(\sigma) / \sigma) \rightarrow 0$ as $\sigma \rightarrow 0$. The notation $O(\sigma)$ was used in the conventional manner.

2. Equation (4) on page 887 should read (using $o(\sigma) \rightarrow 0$ as $\sigma \rightarrow 0$ ):

$$
\hat{K}_{\sigma} \leqslant K-(1-o(\sigma)) I(\Omega ; \sigma) \sigma+\sigma o(\sigma) \text { as } \sigma \rightarrow 0+\text {. }
$$

3. Following Eqn. (9) on p. 889, the function $W_{\tau, \delta}(p)$ should be defined by $W_{\tau, \delta}(p)=$ $\max \left\{\left(\left(U_{\tau}(p)-U_{\tau}(q)\right) / \delta\right): q \in \Omega_{\tau},|p-q| \leqslant \delta\right\}$, and $\delta$ should be $2 \delta$ in the expression defining $M_{\tau, \delta}$.

4. What we have termed the 'angular variation' of a curve on p. 893 is usually called the total curvature, see [2, Section 26].

\section{References}

[1] A. Acker, Free Boundary Optimization-a Constructive Iterative Method, Z. angew. Math. Phys. 30, $885-900(1979)$.

[2] J. C. C. Nitsche, Vorlesungen über Minimalflächen, Springer Verlag, Berlin (1975),

Bevorstehende Internationale Tagungen - List of Forthcoming International Meetings -

Prochaines conférences internationales

Fourth GAMM Conference on Numerical Methods in Fluid Mechanics to be held at ENSTA, Paris (France), October 7-9, 1981.

The GAMM Committee for Numerical Methods in Fluid Mechanics announces its fourth conference organized in cooperation with the Office National d'Etudes et de Recherches Aérospatiales (ONÉRA). The conference, which will be held at the "Ecole Nationale Supérieure de Techniques Avancées" (ENSTA) in Paris, will be concerned with the theory and the application of numerical methods in fluid mechanics (aerodynamics, meteorology, astrophysics, oceanography, biology, nuclear reactor technology, etc.).

Deadline for abstracts (one page, two pages at maximum) is March 15, 1981. The proceedings of the conference will be published by the Vieweg Verlag in the series "Notes on Numerical Fluid Mechanics".

Further information and registration forms can be obtained from: Henri Viviand, Chairman GAMM Conference, ONÉRA, 29, Av. de la Division Leclerc, F-92320 Chatillon, France. 